\title{
A Biochemical analysis demonstrates that the BRCA1 intronic variant IVS10-2A $\rightarrow C$ is a mutation
}

Received: 14 March 2003 / Accepted: 19 May 2003 / Published online: 22 July 2003

(C) The Japan Society of Human Genetics and Springer-Verlag 2003

\begin{abstract}
Sequence analysis of cDNA from an asymptomatic patient belonging to a high-risk breast cancer family carrying the genetic variant $B R C A 1$ IVS10$2 \mathrm{~A} \rightarrow \mathrm{C}$ revealed that functional $B R C A 1 \mathrm{mRNA}$ was derived from only one of the patient's chromosomes. The other chromosome produced an aberrant RNA splicing transcript that deleted exon 11. Analysis of the patient's genomic DNA demonstrated that the chromosome producing the non-functional mRNA carried the genotype BRCA1 IVS10-2A $\rightarrow \mathrm{C}$. This transversion disrupts a highly conserved base in the consensus splice acceptor motif. These results support the conclusion that $B R C A 1$ IVS10-2A $\rightarrow \mathrm{C}$ is a mutation that confers predisposition to breast and ovarian cancer.
\end{abstract}

Keywords $B R C A 1 \cdot$ Splice $\cdot$ Mutation · Intron · Breast cancer $\cdot$ mRNA

\section{Introduction}

Mutations in the tumor suppressor gene $B R C A 1$ confer an increased risk for the development of breast and ovarian cancer. BRCAl is located in a $100-\mathrm{kb}$ region of chromosome 17 (Miki et al. 1994). More than 1,200 genetic variants dispersed throughout $B R C A 1$ have been

J. C. Keaton · D. R. Nielsen · B. C. Hendrickson · M. T. Pyne B. E. Ward · T. Scholl $(\square)$

Technology Development, Myriad Genetic Laboratories, 320

Wakara Way, Salt Lake City, UT 84108, USA

E-mail: tscholl@myriad.com

Tel.: + 1-801-5841126

Fax: + 1-801-5841190

L. Scheuer

Memorial Sloan-Kettering Cancer Center, New York, NY USA

A. R. Brothman

Departments of Pediatrics and Human Genetics,

University of Utah School of Medicine,

Salt Lake City, UT, USA identified. Over 50 of these variants occur within introns or within splice consensus sites and may therefore interfere with RNA processing. The majority of these variants are of unknown clinical significance.

The characterization of genetic variants that do not obviously disrupt an open reading frame can be complicated. Genetic approaches have identified relationships between the occurrence of such variants and an increased risk for cancer (Friedman et al. 1994). Variants that impact splicing lend themselves to biochemical analysis because it is possible to detect their effects on RNA processing. Many splicing mutations reside within consensus sequences in introns and are removed during transcript processing. Therefore, the analysis of these mutations is complicated because the variant itself cannot be used as a marker to differentiate between normal alternative and aberrant splicing products. This determination can be facilitated if specimens contain additional coding region polymorphisms that permit the contribution by both chromosomes to any RNA species to be evaluated.

\section{Subjects and methods}

Subject

The patient described here is 31 years old and has not been diagnosed with cancer but has a family history of breast cancer that includes a first-degree relative diagnosed with breast cancer at age 38 and a second-degree relative diagnosed with breast cancer at age 46 (Fig. 1).

The genetic variant IVS10-2A $\rightarrow \mathrm{C}$ was identified through clinical full-sequence analysis of $B R C A 1$ and $B R C A 2$ (BRACAnalysis, Myriad Genetic Laboratories, Salt Lake City, Utah). The test revealed the intronic variant IVS10-2A $\rightarrow \mathrm{C}$ in $B R C A 1$ and two missense polymorphisms at residues 694 (serine AGC/AGT) and 1,436 (serine TCT/TCC), which have been characterized as clinically insignificant. The patient was informed of the uncertain clinical significance of the intronic variant through genetic counseling and elected to participate in a research protocol designed to further characterize the variant. Unfortunately, both relatives of the patient that were diagnosed with cancer declined to submit blood samples for analysis. 
Fig. 1 Patient pedigree. The patient (indicated with arrow) was 31 years old and had not been diagnosed with cancer. One first-degree and one second-degree relative had previously been diagnosed with breast cancer at 38 and 46 years old, respectively. The numbers 3 and 7 within boxes indicate the number of additional male children in that family

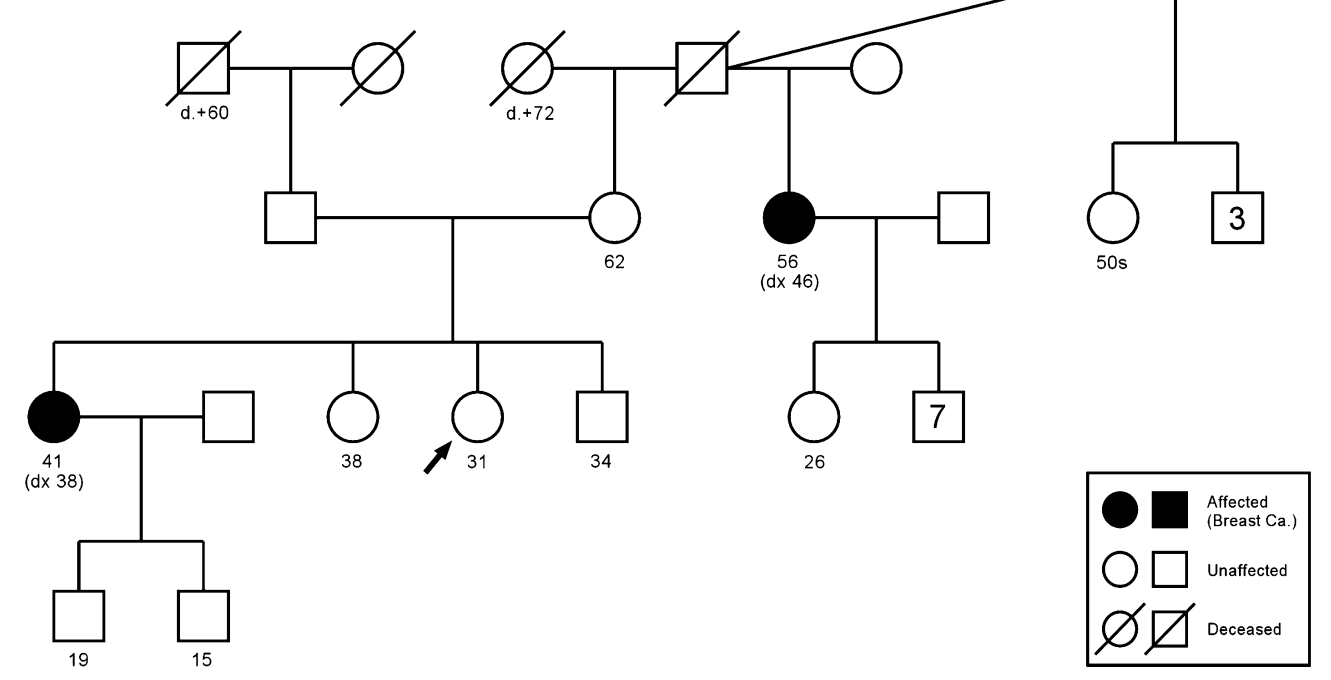

Nucleic acid techniques

Commercially available kits were used to extract RNA from peripheral blood lymphocytes, which was subsequently used for cDNA synthesis (RNAeasy, Qiagen, Chatsworth, Calif. and Superscript Preamplification Kit, Gibco BRL, Gaithersburg, Md.). The primers used in these experiments contained the following designations and gene-specific sequences (all numeric base-pair designations conform to GenBank submission U14680, except for primer $11 \mathrm{~F} 3$, which anneals to genomic DNA and conforms to GenBank submission L78833):

6F 5'-GTGCTTTTCAGCTTGACACAGGTTT-3' (bases 391-415) $11 F 1$ 5'-TAGTAGTCAGTAGAAATCTAAGCCCACC-3' (bases 1993-2020)

$11 F 2$ 5'-ATCAAGAAGAGCAAAGCATGGATTCAA-3' (bases 4183-4209)

$11 F 3$ 5'-GTTAGTTTCTCTAATATAGCCAGTTGG-3' (bases 33756-33782)

$11 R 1$ 5'-ACTGACTCACACATTTATTTGGTTCTG-3' (bases 2532-2506)

$11 R 2$ 5'-TACTGGGTTGATGATGTTCAGTATTTGTTA-3' (bases 846-817)

$12 F$ 5'-GGGCTATCCTCTCAGAGTGACAT-3' (bases 42694291)

$12 R$ 5'-AGCAGTCTTCAGAGACGCTTGTTT-3' (bases 42664243)

13-14R1 5'-TGAAGTTAATACTGCTTTTTCTGATGTGCTT-3' (bases 4490-4460)

$15 R$ 5'-CAACTGTGCATGTACCACCTATCATCTA-3' (bases 4657-4630)

16R 5'-TGACCCTTTCTGTTGAAGCTGTCAA-3' (bases 50585034)

S694-AGC-R 5'-ACTTCAGCTCTGGGAAAGTATCGC-3' (bases 2223-2200)

S694-AGT-R 5'-CTTCAGCTCTGGGAAAGTATCAC-3' (bases 2222-2200)

All primers carried M13 tails with the sequences 5'-GTTTTCCCAGTCACGACG-3' for forward primers (designated " $F$ ") and 5'-AGGAAACAGCTATGACCAT-3' for reverse primers (designated "R"). The allele-specific primers, S694-AGT-R and S694-AGC-R, contained thioate derivative base analogs at the two most $3^{\prime}$ residues.

All polymerase chain reactions (PCR) employed TaqPlus Long polymerase in the accompanying high salt buffer (Stratagene, La Jolla, Calif.) with primers at $1 \mathrm{pg}$ each and target genomic DNA or
cDNA at a concentration of approximately $20 \mathrm{ng}$ and $10 \mathrm{ng}$, respectively. Following the initial denaturation step, thermal cycling proceeded using 35 cycles at $94{ }^{\circ} \mathrm{C}$ for $20 \mathrm{~s}, 62^{\circ} \mathrm{C}$ for $30 \mathrm{~s}$, and $72{ }^{\circ} \mathrm{C}$ for $1-3 \mathrm{~min}$, depending on product length.

Nucleotide sequencing employed dye-primer chemistry (ABI PRISM BigDye Primer Sequencing, Applied Biosystems, Foster City, Calif.) detected on automated instruments (ABI PRISM 377 DNA Sequencer Model 377, Applied Biosystems). The genotypes identified during the clinical test used for this study were confirmed in the patient and in the control samples prior to transcript analysis.

\section{Results}

Analysis of RNA transcripts

RNA splicing was evaluated by amplifying regions of $B R C A 1$ from cDNA derived from the patient and from a control sample that shared the polymorphisms S694S and S1436S. Polymerase chain reactions were designed to generate fragments that contained the exon junctions most likely affected by the variant IVS10-2A $\rightarrow \mathrm{C}$ and the coding region polymorphisms S694S and S1436S. The fragments produced in these reactions were separated on agarose gels, and the patient and control samples were compared. Some fragments were excised for further analysis. These excised fragments served as targets for additional PCR-based sequencing reactions that could confirm the primary structure of the transcripts at the splice junctions and also determine the contribution from both chromosomes to any RNA species by assessing the nucleotide sequence at the polymorphic sites. The fragments generated for this analysis are depicted schematically (Fig. 2).

Fragments were generated by PCR to examine the $B R C A 1$ message upstream from exon 11 using the primer pair $6 \mathrm{~F}$ and $11 \mathrm{R} 1$. The sequences of these products showed normal splice junctions for exons 9 through 11 in both the patient and control (primer set $6 \mathrm{~F}$ and 11R2, 


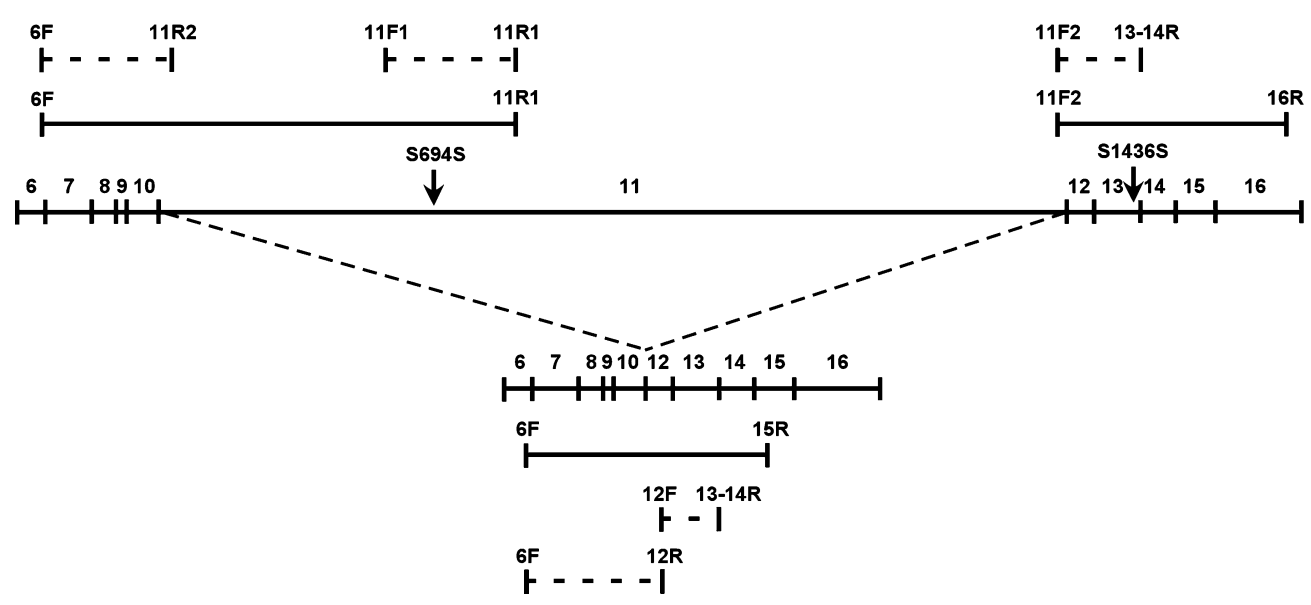

Fig. 2 Fragments generated for cDNA analysis. Both the normal mRNA and the aberrant mRNA splicing product that deletes exon 11 are depicted schematically. PCR fragments generated for these analyses are shown, and the primers used for amplification are indicated for each fragment. Arrows indicate the positions of the polymorphisms at codons 694 (serine AGC/AGT) and 1,436 (serine TCT/TCC). Fragments adjacent to the mRNA schematics were amplified from cDNA and fractionated and excised from agarose gels. Fragments one line removed from the primary amplification reaction (horizontal dashed lines) were generated by secondary amplification of the respective gel-purified fragment and sequenced

Fig. 3a). In the patient, however, this normal transcript was derived solely from the chromosome encoding AGT at residue 694, while the same transcript was derived from both chromosomes in the control sample (primer set $11 \mathrm{~F} 1$ and $11 \mathrm{R} 1$, Fig. $3 \mathrm{~b}$ ). This result, which indicates that the patient has lost the functional transcript from one chromosome, was confirmed when the polymorphism at residue 1,436 was evaluated by sequencing. Gelpurified fragments from PCR using the primer pair 11F2 and $16 \mathrm{R}$ were sequenced using the primer set $11 \mathrm{~F} 2$ and 13-14R. The results of these sequencing data demonstrated that the functional mRNA in the patient was produced only from the chromosome encoding TCC at residue 1,436, while the control sample was heterozygous at that codon (Fig. 3c, d). Furthermore, the two bases detected on the transcripts in the patient at residues 694 and 1,436 occur together on a common haplotype (Shattuck-Eidens et al. 1997). These results support the conclusion that the normal mRNA for BRCAl is produced from only one chromosome in the patient.

Mutations within splice acceptor sites commonly result in transcript processing errors that delete the subsequent exon (Berget 1995; Zhang 1998). The patient and control cDNA samples were amplified with the primer pair $6 \mathrm{~F}$ and $15 \mathrm{R}$ to determine if transcripts were processed that delete exon 11 . When the products from these reactions were visualized on agarose gels, one band was common to both the patient and the control, while three additional fragments were detected from the patient (Fig. 4). These fragments were excised from the gel, amplified and sequenced using the primer pairs $6 \mathrm{~F} / 12 \mathrm{R}$ and $12 \mathrm{~F} / 13-14 \mathrm{R}$. The common fragment is a normal alternative product that results from the use of a cryptic splice donor site that causes the deletion of the 3,309 carboxy-terminal nucleotides of exon 11 from the transcript. The longest of the three bands unique to the patient results from the complete deletion of exon 11 . Furthermore, this splicing product is produced from the chromosome that encodes S1436/TCT (Fig. 5). The two shorter fragments that were detected in the patient are also produced from only the chromosome encoding S1436/TCT and are caused by the deletions of exons 10 and 11 and exons 9 through 11 . The position of the IVS10-2A $\rightarrow \mathrm{C}$ variant within the splice acceptor site and the detection of shorter fragments from the chromosome generating non-functional mRNA suggest that this variant causes aberrant RNA splicing and produces multiple mutant transcripts that delete exon 11. The smaller transcripts appear to be compound deletions of exon 11 caused by IVS10-2A $\rightarrow \mathrm{C}$ with normal alternative products that delete either exon 10 or exons 9 and 10 (Lu et al. 1996; Miki et al. 1994).

The intronic variant IVS10-2A $\rightarrow \mathrm{C}$ was associated with the chromosome that encodes 694/AGT by amplifying the patient and control genomic DNA using the forward primer $11 \mathrm{~F} 3$ with the reverse primer $11 \mathrm{R} 1$ and the allele-specific reverse primers S694-AGC-R and S694-AGT-R. In the patient, the sequence of these fragments shows the heterozygous base pair $\mathrm{A} / \mathrm{C}$ at IVS10-2A $\rightarrow \mathrm{C}$ when amplified with primer 11R1, while primer S694-AGC-R was associated with cytosine and primer S694-AGT-R was associated with adenine at that position (Fig. 6). This result demonstrates that the intronic variant resides on the chromosome that does not produce normal mRNA, but instead contributes to an aberrant splicing product that deletes exon 11 .

\section{Discussion}

Three separate results corroborate to support the conclusion that BRCAI IVS10-2A $\rightarrow \mathrm{C}$ is a deleterious mutation that induces aberrant splicing that deletes exon 11 and confers risk for hereditary breast cancer. First, the normal mRNA for $B R C A 1$ is derived from only one chromosome in the patient. Second, an 
Exon Junctions

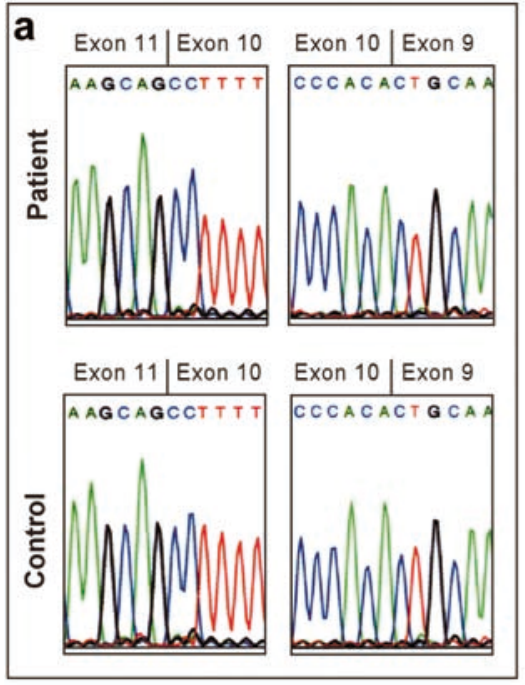

\section{Exon Junctions}

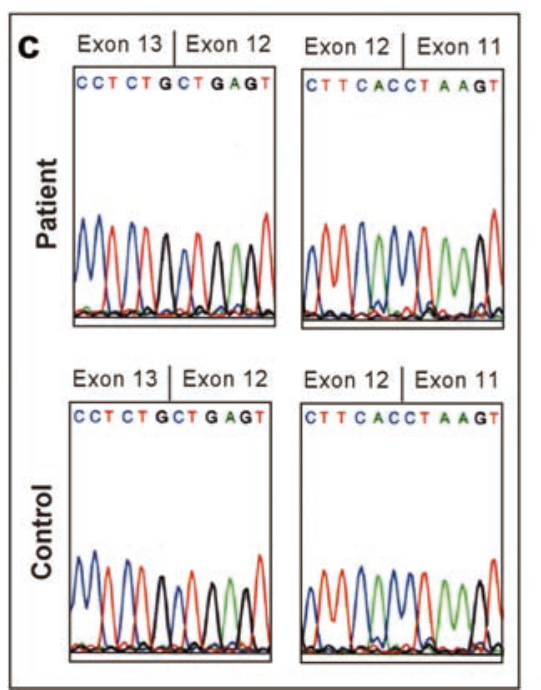

\section{Polymorphism}

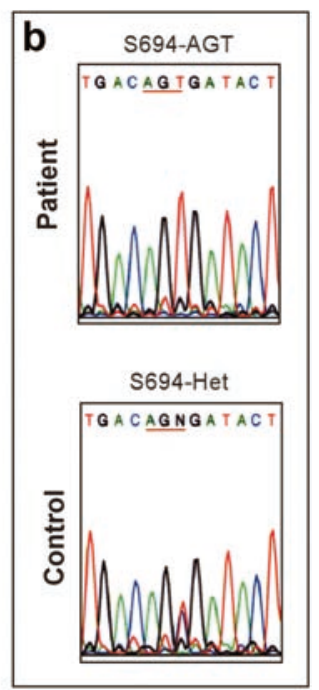

Polymorphism

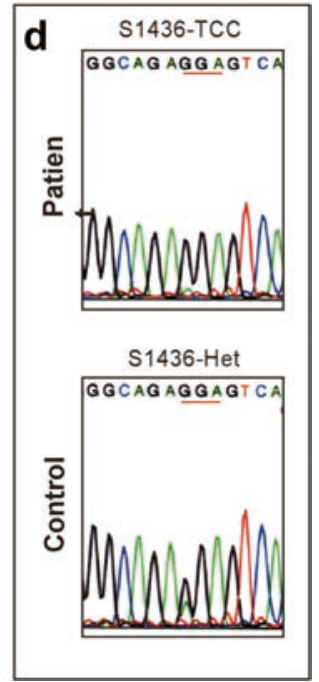

Fig. 3a-d Sequence analysis of normal BRCAl mRNA from patient and control. Portions of fluorescent sequence data (extracted with ABI Prism sequencing analysis software version 2.1.1) surrounding codons 694 and 1,436 and the exon junctions that would likely be affected by the variant are shown. The reverse strand is used for all traces except for the polymorphism at codon 694. These sequencing reactions were performed from primary PCR reactions using primers $6 \mathrm{~F}$ and $11 \mathrm{R} 1$ for the $5^{\prime}$ region from exon $11(\mathbf{a}, \mathbf{b})$ and primers $11 \mathrm{~F} 2$ and $16 \mathrm{R}$ for the $3^{\prime}$ region from exon $11(\mathbf{c}, \mathbf{d})$. The fragments were excised from agarose gels and sequenced. Both the patient and control samples showed normal splice junctions for exons $9-11$ and $11-13$, using the primer sets $6 \mathrm{~F} /$ $11 \mathrm{R} 2$ and $11 \mathrm{~F} 2 / 13-14 \mathrm{R}$, respectively $(\mathbf{a}, \mathbf{c})$. The sequence of the functional mRNA transcript from the patient demonstrates that it was derived solely from the allele that carries the non-consensus codons 694 (AGT) using primers $11 \mathrm{~F} 1$ and $11 \mathrm{R} 1$ and 1436 (TCC) using primers $11 \mathrm{~F} 2$ and $13-14 \mathrm{R}(\mathbf{b}$, d). In panel d, although the analysis software called a "G" at the position for the polymorphism S1436S in the control sample, the sequence trace clearly exhibits the heterozygous bases $\mathrm{A} / \mathrm{G}$

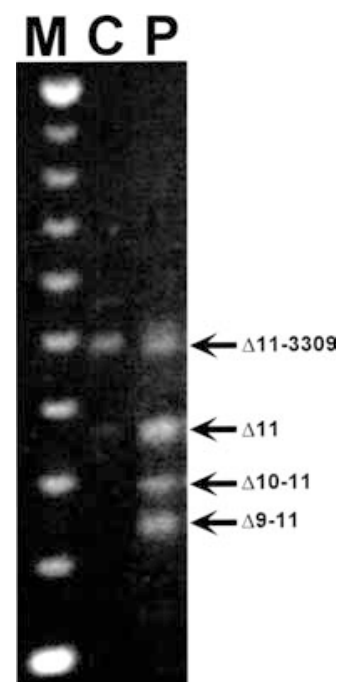

Fig. 4 Detection of additional RNA splice products in the patient. PCR fragments generated with the primer pair $6 \mathrm{~F}$ and $15 \mathrm{R}$ from cDNA were separated by agarose gel electrophoresis $[M 100-b p$ ladder (fragments 1,500-600 shown), $C$ control sample, $P$ patient sample]. Three bands unique to the patient were detected. These fragments represent aberrant RNA splicing products that result from IVS10-2A $\rightarrow \mathrm{C}$ (see text). The band common to the patient and control is a normal alternative product that is produced by a cryptic donor site within exon 11 (see text)

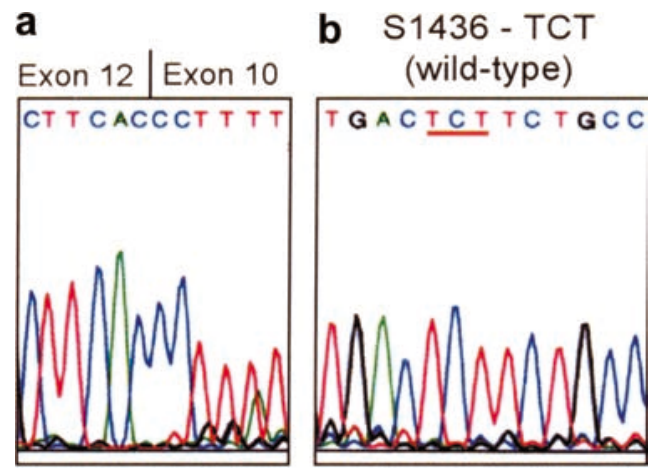

Fig. 5a, b Sequence analysis of an aberrant RNA transcript in the patient. These traces represent portions of fluorescent sequence data from the patient's cDNA. The largest of three fragments that amplified from only the patient using primers $6 \mathrm{~F}$ and $15 \mathrm{R}$ was excised and sequenced. a This sequence reveals the deletion of exon 11 using primers $6 \mathrm{~F}$ and $12 \mathrm{R}$. b The deletion occurs on the chromosome encoding residue 1436 with TCT using primers $12 \mathrm{~F}$ and 13-14R. (Sense strand shown for the polymorphic codon, and the reverse strand shown for the aberrant exon junction)

aberrant transcript that deletes exon 11 is produced solely or in great excess from the other chromosome. Also, the structure of this RNA is consistent with expectations for exon skipping induced by a mutation in a splice acceptor site. Third, the variant IVS10-2A $\rightarrow \mathrm{C}$ was physically associated to the chromosome that produces the aberrant RNA by allele-specific PCR. The removal 


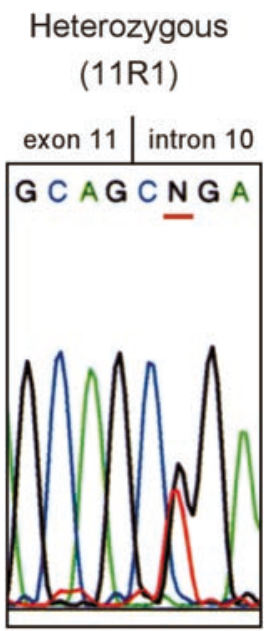

a
IVS10-2A (S694-AGT-R)

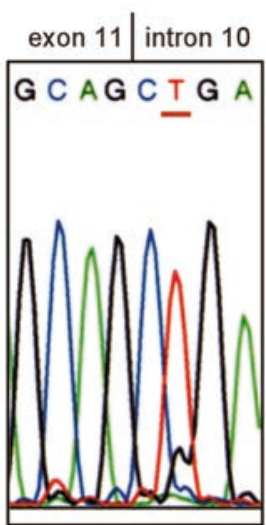

b
IVS10-2C (S694-AGC-R)

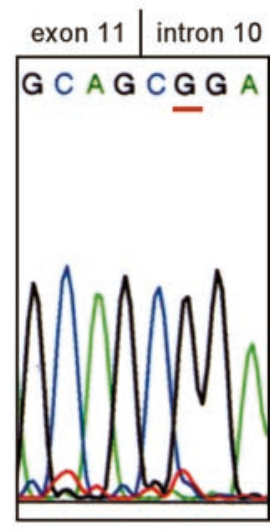

C
Fig. 6a-c Association of IVS10-2A $>C$ to the chromosome encoding S694 with AGC. Genomic DNA from the patient was amplified using primer $11 \mathrm{~F} 3$ in combination with the reverse primer $11 \mathrm{R} 1$ or the allele-specific primers S694-ACG-R and S694-AGT-R (polymorphic base one nucleotide removed from the $3^{\prime}$ end of the primer, see Materials and methods). a The heterozygous base at IVS10-2A $\rightarrow \mathrm{C}$ is shown using the reverse primer $11 \mathrm{R} 1$. b Using the allele-specific reverse primer S694-AGT-R, only the base IVS10-2A is shown. c Using the allele-specific reverse primer S694-AGC-R, only the mutant base IVS-2C is shown

of exon 11 produces transcripts that resume the normal reading frame following the deletion of 1,143 residues ( $B R C A 1$ contains 1,863 amino acids). This same deletion in mouse models abrogates BRCAl function (Xu et al. 1999a, 1999b).

There is growing acceptance of the utility for genetic testing to assess patients' predisposition to disease. It is essential to develop methods to determine the clinical significance of genetic variants in patients that are asymptomatic or where samples from other family members are unavailable. Here, a method for conclusive biochemical analysis of variants with the potential to impact RNA splicing is demonstrated. This method has previously produced definitive results by identifying splicing mutations in diagnosed cancer patients (Pyne, et al. 1999, 2000; Scholl et al. 1999). In this instance, the formal demonstration of this RNA splicing mutation will occur when the relationship between this variant and the occurrence of breast or ovarian cancer can be established clinically.

\section{References}

Berget SM (1995) Exon recognition in vertebrate splicing. J Biol Chem 27:2411-2414

Friedman LS, Ostermeyer EA, Szabo CI, Dowd P, Lynch ED, Rowell SE, King MC (1994) Confirmation of BRCAl by analysis of germline mutations linked to breast and ovarian cancer in families. Nat Genet 8:399-404

Lu M, Conzen SD, Cole CN, Arrick BA (1996) Characterization of functional messenger RNA splice variants of $B R C A 1$ expressed in nonmalignant and tumor-derived breast cells. Cancer Res 56:4578-4581

Pyne MT, Pruss D, Ward BE, Scholl T (1999) A characterization of genetic variants in BRCAl intron 8 identifies a mutation and a polymorphism. Mutat Res 406:101-107

Pyne MT, Brothman AR, Ward B, Pruss D, Hendrickson BC, Scholl T (2000) The BRCA2 genetic variant IVS7 $+2 \mathrm{~T} \rightarrow \mathrm{G}$ is a mutation. J Hum Genet 45:351-357

Miki Y, Swensen J, Shattuck-Eidens D, Futreal A, Harshman K, Tavtigian S, Liu Q, Cochran C, Bennett LM, Ding W, Bell R, Rosenthal J, Hussey C, Tran T, McClure M, Frye C, Hattier T, Phelps R, Haugen-Strano A, Katcher H, Yakumo K, Gholami Z, Shaffer D, Stone S, Bayer S, Wray C, Bogden R, Dayananth P, Ward J, Tonin P, Narod S, Bristow PK, Norris FH, Helvering L, Morrison P, Rosteck P, Lai M, Barrett JC, Lewis C, Neuhausen S, Cannon-Albright L, Goldgar D, Wiseman R, Kamb A, Skolnick MH (1994) A strong candidate for the breast and ovarian cancer susceptibility gene $B R C A 1$. Science 266:6671

Shattuck-Eidens D, Oliphant A, McClure M, McBride C, Gupte J, Rubano T, Pruss D, Tavtigian SV, Teng DH Adey N, Staebell M, Gumpper K, Lundstrom R, Hulick M, Kelly M, Holmen J, Lingenfelter B, Manley S, Fujimura F, Luce M, Ward B, Cannon-Albright L, Steele L, Offit K, Gilewski T, Norton L, Brown K, Schulz C, Hampel H, Schluger A, Giulotto E, Zoli W, Ravaioli A, Nevanlinna H, Pyrhonen S, Rowley P, Loader S, Osborne MP, Daly M, Tepler I, Weinstein PL, Scalia JL, Michaelson R, Scott RJ, Radice P, Pierotti MA, Garber JE, Isaacs C, Peshkin B, Lippman ME, Dosik MH, Caligo MA, Greenstein RM, Pilarski R, Weber B, Burgemeister R, Frank TS, Skolnick MH, Thomas A (1997) BRCA1 sequence analysis in women at high risk for susceptibility mutations: risk factor analysis and implications for genetic testing. JAMA 278:12421250

Scholl T, Pyne MT, Ward BE (1999) BRCA1 IVS16 +6T $\rightarrow$ C causes aberrant splicing and the activation of a cryptic donor site. Am J Med Genet 85:113-116

Xu X, Wagner K-U, Larson D, Weaver Z, Li C, Ried T, Hennighausen L, Wynshaw-Boris A, Deng C-X (1999a) Conditional mutation of $B R C A 1$ in mammary epithelial cells results in blunted ductal morphogenesis and tumour formation. Nat Genet 22:37-43

Xu X, Weaver Z, Linke SP, Li C, Gotay J, Wang X-W, Harris CC, Ried T, Deng C-X (1999b) Centrosome amplification and a defective G2-M cell cycle checkpoint induce genetic instability in BRCA1 exon 11 isoform-deficient cells. Mol. Cell 3:389-395

Zhang MQ (1998) Statistical features of human exons and their flanking regions. Hum Mol Genet 7:919-932 